\title{
MHD Fluid Flow of an Exponentially Varying Plasma Density in a Radiating and Slowly Rotating Hot Sphere
}

\author{
B. S. Tuduo ${ }^{1, ~}$, T. M. Abbey ${ }^{1}$, K. D. Alagoa $^{2}$ \\ ${ }^{1}$ Department of Physics, University of Port Harcourt, Port Harcourt, Nigeria \\ ${ }^{2}$ Department of Physics, Niger Delta University, Amassoma, Nigeria
}

Email address:

bieseb4prom@gmail.com (B. S. Tuduo), tamunoimi.abbey@uniport.edu.ng (T. M. Abbey), dalagoa@yahoo.com (K. D. Alagoa)

${ }^{*}$ Corresponding author

\section{To cite this article:}

B. S. Tuduo, T. M. Abbey, K. D. Alagoa. MHD Fluid Flow of an Exponentially Varying Plasma Density in a Radiating and Slowly Rotating Hot Sphere. International Journal of Astrophysics and Space Science. Vol. 6, No. 1, 2018, pp. 18-27. doi: 10.11648/j.ijass.20180601.12

Received: June 15, 2017; Accepted: July 6, 2017; Published: February 11, 2018

\begin{abstract}
The study presents the effect of density variation on the flow structure of a plasma gas in a slowly rotating and radiating hot sphere. The problem which is solved by general perturbation method shows that the plasma temperature decreases to a minimum at a radial distance of 1.4 solar radii and then increased to a maximum value at a radial distance of 3.5 solar radii, for various radiation parameters, $N^{2}$. The sudden increase in temperature profile when the radial distance is 1.4 solar radii, indicates the heating up of the upper regions of the solar atmosphere.
\end{abstract}

Keywords: MHD Flow, Exponentially Varying Plasma Density, Radiating Hot Sphere

\section{Introduction}

MHD flows have found useful applications in several fields of study such as engineering (as in the case of MHD power generation, plasma confinement, liquid metal cooling of nuclear reactors, etc), medicine, geophysics and as well as astrophysics, where magnetic field is seen to play a dominant role in the activities of stars and their formation [1 - 6, 35]. Our Sun which is a star in the Milky-way galaxy with a surface temperature of $a \times 10^{6}$ degree Kelvin, has the ability to replenish the huge amount of energy lost, in the form of radiation, to its surrounding through nuclear fusion at the core. In the literature, the studies of MHD flows abound, but in most of them the plasma density is usually considered constant and the effect of compressibility assumed to be zero. According to [7], ignoring the effect of compressibility in such flow models is rather dangerous.

This is because experiment indicates that free convection motion in a flow regime is usually caused by changes in the local density due to variations in the hydrostatic pressure resulting in isotropic acceleration of the fluid.

In this regards, several reports in the literature have highlighted the import of density in the study of astrophysical plasma. For instance, [8] calculated the variation of the brightness and polarization of the k-corona under the assumption of isothermal corona with a hydrostatic description of density. Also, [9] emphasized the possible deduction of hydrostatic coronal temperature from the coronal electron density and the relation of the coronal white light, which is due to scattering, to free electron density. [10] and [11] respectively described the distribution of the inner coronal electron densities as an exponential function of radial distance from the solar core to obtain the coronal brightness.

Similarly, the astrophysical data presented by [12] show that the plasma density within the solar interior and its atmosphere changes from one layer to another as one moves away from the core. To this end [13] presented a model and show that the plasma density in the solar sphere has an exponential dependent function.

In all the studies above, none have included the effect of radiation and heat transfer on the flow model. This study therefore, is to address the effect of plasma density variation on the flow structure of an ionized gas in the present of radiation and convective heat transfer in a slowly rotating hot sphere.

The study is divided into four main sections. Section 2 presents the physics and the mathematical formulations of the problem, leading to the governing equations of the problem. On the other hand, sections 3 and 4, respectively handle the method of solutions and the analysis, and the discussion of the various results of the problem with respect to some realistic values of the flow parameters. 


\section{Problem Formulations}

The problem considers the flow of a plasma gas whose density varies radially in a slowly rotating and radiating hot sphere. The surface temperature of the solar sphere according to observations is $T_{s}=a \times 10^{6}$ degree Kelvin, where $a$ is a constant integer. Several findings in the literature and astrophysical observations show that unlike pulsars, the Sun rotates slowly about the azimuthal with an angular velocity, $\Omega_{0}$ such that its magnetic Reynolds number $R_{m}$ is far less than one (i.e., $R_{m}<<1$ ). This implies that the induced

$$
\rho(r)=98.8 A v_{o} \cdot\left(\rho_{\text {mass }}\right)\left[10^{\exp [-11.1 r+o .15 / r]}+10^{\exp [-11.1 r /(1+0.02 / r)]}\right]
$$

where, $A v_{o}$ and $\rho_{\text {mass }}$ are the Avogadros number and proton mass, respectively.

Also, due to nuclear processes within the core, the Sun as a star has a core temperature in the order of $10^{7}$ degree Kelvin in relation to its surface with temperature of just about 5,800 degree Kelvin. That is, the core temperature $T_{c}$ is far greater than the surface temperature $T_{s}\left(T_{c} \gg T_{s}\right)$. This is responsible for radiative heat transfer.

If, therefore, $\rho^{\prime}\left(r^{\prime}\right)$ is the density of the plasma as a function of $r^{\prime}$, and $V^{\prime}=\left(u^{\prime}, v^{\prime}, w^{\prime}\right)$ are respectively the velocity components in the orthogonal $\left(r^{\prime}, \theta, \varphi\right)$ directions of the spherical coordinate system; $p^{\prime}$, the pressure; $T^{\prime}$, the temperature; $q_{r}^{\prime}$, the radiative heat transfer flux vector; $\mu$, the dynamical viscosity; $\chi$, the permeability; $C_{p}$, the specific heat capacity at constant pressure; $\kappa$, the thermal conductivity; $\sigma$, the Stefan-Boltzmann constant; $\gamma$, thermal expansivity coefficient; $\alpha_{r}$, the absorption coefficient and $T_{\infty}$, is the temperature of the medium at equilibrium with $T_{w}$ $=T_{s}$, the wall or surface temperature which is kept constant, then the mathematical equations governing the flow of the plasma in the stratified density layers of the solar body, considering the Boussinesq approximation and following the method adopted by [1, 3, 17 - 18] can be presented as follows;

$$
\begin{gathered}
\nabla \bullet\left(\rho^{\prime} V^{\prime}\right)=0 \\
\rho^{\prime}\left[\left(V^{\prime} \bullet \nabla\right) V^{\prime}\right]=-\nabla \bullet P^{\prime}+\mu \nabla^{2} V^{\prime}-\frac{\mu}{\chi} V^{\prime}-\sigma B_{0}^{2} V^{\prime}+ \\
\rho^{\prime} \beta g\left(T^{\prime}-T_{w}\right) \\
\rho^{\prime} C_{p}\left[(V \bullet \nabla) T^{\prime}\right]=K \nabla^{2} T^{\prime}-\nabla q_{r}^{\prime}
\end{gathered}
$$

and

$$
\nabla \bullet \nabla q_{r}^{\prime}-3 \sigma \alpha^{2} q_{r}^{\prime}-4 \sigma \alpha T^{\prime 3} \nabla T^{\prime}=0
$$

where, the operator $\nabla$ has its usual meaning and $g$ is the acceleration due to gravity. Equations (1) to (4) are respectively the continuity, force, energy and the generalized Rosseline radiative heat transfer flux [19]. These equations, in other words, are the expressions for the conservation of magnetic field $\mathrm{B}^{\prime}$ can be neglected and the flow is seen to be influenced by the applied magnetic field, $B_{0}$, which manifest as a result of the dynamo action within the interior of the astrophysical object. Similarly, the analysis of the astrophysical data presented by $[12,14-16]$ reveal that the plasma density distribution in the Sun is as follows: (the core $\left(1.6 \times 10^{5} \mathrm{~kg} / \mathrm{m}^{3}\right)$, the radiative zone $\left(2 \times 10^{-3} \mathrm{~kg} / \mathrm{m}^{3}\right)$, the convective zone $\left(5 \times 10^{-4} \mathrm{~kg} / \mathrm{m}^{3}\right)$, the photosphere $\left(8 \times 10^{-5}\right.$ $\left.\mathrm{kg} / \mathrm{m}^{3}\right)$, the chromospheres $\left(5 \times 10^{-6} \mathrm{~kg} / \mathrm{m}^{3}\right)$ and the corona $\left.\left(1 \times 10^{-11} \mathrm{~kg} / \mathrm{m}^{3}\right)\right)$.

Following these, [13] indicates that the plasma density distribution in the solar globe can be illustrated as; mass, momentum and energy within the system.

As was observed in the case of [13] and [20], the plasma gases in the intergalactic and interplanetary layers are seen to be rarefied. Such that the optical property, $\alpha_{r}$, of the plasma is far less than one (i.e., $\left.\alpha_{r} \ll 1\right)$. That is, the gas in this region is mostly regarded as optically thin. Hence, the generalized Rosseline radiative heat flux integro-differential equation for the optically thin limit can be expressed as;

$$
\nabla q_{r}^{\prime}=16 \alpha \sigma\left(T^{\prime 4}-T_{\infty}^{4}\right)
$$

Furthermore, from the statistical data presented by [13], as well as the models of [21] and [22], the temperature difference between adjacent layers of the plasma is not much compared to each other, thus;

$$
T^{\prime}=T_{\infty}+\phi
$$

where, $\phi$ is a small temperature correction factor, such that, $O\left(T^{\prime}\right) \gg>>>O\left(T_{\infty}\right)$, then equation (5), the heat transfer flux vector equation reduced to;

$$
\nabla q_{r}^{\prime}=16 \sigma \alpha T_{\infty}^{3}\left(T_{\infty}+\phi\right)
$$

and the heat transfer equation becomes;

$$
\rho^{\prime} C_{p}\left[\left(V^{\prime} \bullet \nabla\right) \phi\right]=K \nabla^{2} \phi-16 \sigma \alpha T_{\infty}^{3} \phi
$$

Equations (1), (2) and (8) shall be solved subject to the following boundary conditions;

$$
T^{\prime}=T_{c} \text {, and } u^{\prime}, v^{\prime}, w^{\prime}=0 \text { on } \mathrm{r}=0.25 R_{\otimes}(\text { near the core })
$$
and

$T^{\prime}=T_{w}$, and $u^{\prime}, v^{\prime}, w^{\prime}=0$ on $\mathrm{r}=1.0 R_{\otimes}$ (the surface); where, $R_{\circledast}$ is the solar radius.

Now, introducing the following non-dimensional relations;

$$
r=\frac{r^{\prime}}{R_{0}},(u, v, w)=\frac{\left(u^{\prime}, v^{\prime}, w^{\prime}\right)}{\Omega_{0} R_{0}}, \rho(r)=\frac{\rho^{\prime}(r)}{\rho_{0}}, \Theta=\frac{\phi-T_{\infty}}{T_{w}-T_{\infty}},
$$

and eliminating the pressure gradients in the $r$ and $\theta$ directions, Equations (1), (2) and (8) in the spherical coordinate system $(r, \theta, \varphi)$ becomes; 


$$
\begin{gathered}
\rho \frac{\partial u}{\partial r}+\frac{2 \rho u}{r}+u \frac{\partial \rho}{\partial r}+\frac{\rho v}{r} \cot \theta+\frac{\rho}{r} \frac{\partial v}{\partial \theta}+\frac{1}{r \sin \theta} \frac{\partial}{\partial \varphi}(\rho w)=0 \\
\operatorname{Re} \rho\left[u \frac{\partial u}{\partial r}+\frac{v}{r} \frac{\partial u}{\partial \theta}+\frac{w}{r \sin \theta} \frac{\partial u}{\partial \varphi}-\frac{v^{2}+w^{2}}{r}\right]=\left[\nabla^{2}-\chi^{2}-M^{2}-\frac{1}{r^{2} \sin ^{2} \theta}\right] u-\frac{2}{r^{2}} \frac{\partial v}{\partial \theta}-\frac{2 v}{r^{2}} \cot \theta-\frac{2}{r^{2} \sin \theta} \frac{\partial w}{\partial \varphi}+\rho G_{r} \Theta \\
\operatorname{Re} \rho\left[\frac{\partial v}{\partial t}+u \frac{\partial v}{\partial r}+\frac{v}{r} \frac{\partial v}{\partial \theta}+\frac{w}{r \sin \theta} \frac{\partial v}{\partial \varphi}+\frac{u v}{r}-\frac{w^{2}}{r} \cot \theta\right]=\left[\nabla^{2}-\chi^{2}-M^{2}-\frac{1}{r^{2} \sin ^{2} \theta}\right] v+\frac{2}{r^{2}} \frac{\partial u}{\partial \theta}-\frac{2 \cos \theta}{r^{2} \sin ^{2} \theta} \frac{\partial w}{\partial \varphi}+\rho G_{r} \Theta \\
\operatorname{Re} \rho\left[u \frac{\partial w}{\partial r}+\frac{v}{r} \frac{\partial u}{\partial \theta}+\frac{w}{r \sin \theta} \frac{\partial w}{\partial \varphi}+\frac{u w}{r}+\frac{v w}{r} \cot \theta\right]=\left[\nabla^{2}-\chi^{2}-M^{2}-\frac{1}{r^{2} \sin ^{2} \theta}\right] w+\frac{2}{r^{2} \sin \theta} \frac{\partial u}{\partial \varphi}+\frac{2 \cos \theta}{r^{2} \sin ^{2} \theta} \frac{\partial v}{\partial \varphi}+\rho G_{r} \Theta \\
\operatorname{Re} \operatorname{Pr} \rho\left[u \frac{\partial \Theta}{\partial r}+\frac{v}{r} \frac{\partial \Theta}{\partial \theta}+\frac{w}{r} \frac{\partial \Theta}{\partial \varphi}\right]=\left(\nabla^{2}-N^{2}\right) \Theta
\end{gathered}
$$

where, $\nabla^{2}=\frac{\partial^{2}}{\partial r^{2}}+\frac{2}{r} \frac{\partial}{\partial r}+\frac{1}{r^{2}} \frac{\partial^{2}}{\partial \theta^{2}}+\frac{\cot \theta}{r^{2}} \frac{\partial}{\partial \theta}+\frac{1}{r^{2} \sin \theta} \frac{\partial^{2}}{\partial \varphi^{2}}$,

$\operatorname{Re}=\frac{\rho_{0} \Omega_{0} R_{0}^{2}}{\mu} ;$ the rotational Reynolds number,

$\chi^{2}=\frac{R_{0}^{2}}{k} ;$ the porosity parameter,

$M^{2}=\frac{\sigma B_{0}^{2} R_{0}^{2}}{\mu} ;$ the magnetic parameter (the Hartmann number),

$G_{r}=\frac{\rho_{0} R_{0}}{\Omega_{0} \mu} \gamma g\left(T^{\prime}-T_{\infty}\right) ;$ the Grashof number,

$\operatorname{Pr}=\frac{\mu C_{p}}{\kappa} ;$ the Prandtl number, and

$N^{2}=\frac{16 \alpha_{r} \sigma R_{0}^{2} T_{\infty}}{\kappa}$; the radiation or heat transfer

parameter.

with,

$\Theta=\Theta_{c}$ and $u, v, w=0$, on $r=0.25 R_{\circledast}$ (near the core)

and,

$\Theta=\Theta_{w}$ and $u, v, w=0$, on $r=1.0 R_{\bigotimes}$ (the surface).

This brings to conclusion the physics and the mathematical formulations of the problem. Next to be considered is the analysis and method of solution.

\section{Method of Solution}

The formulations show that the density of the plasma can be expressed as;

$$
\begin{gathered}
\rho \frac{\partial u_{0}}{\partial r}+\frac{2 \rho}{r} u_{0}+u_{0} \frac{\partial \rho}{\partial r}+\frac{\rho}{r} \frac{\partial v_{0}}{\partial \theta}+\frac{\rho}{r} v_{0} \cot \theta=0 \\
\left(\nabla^{2}-\chi^{2}-M^{2}-\frac{1}{r^{2} \sin ^{2} \theta}\right)\left[\frac{1}{r} \frac{\partial}{\partial r}\left(r v_{0}\right)-\frac{1}{r} \frac{\partial u_{0}}{\partial \theta}\right]+\rho G_{r}\left(\frac{\partial \Theta_{0}}{\partial r}-\frac{\partial \Theta_{0}}{\partial \theta}\right)+G_{r} \Theta_{0}\left(\frac{\partial \rho}{\partial r}+\frac{\rho}{r}\right)=0 \\
\left(\nabla^{2}-\chi^{2}-M^{2}-\frac{1}{r^{2} \sin ^{2} \theta}\right) w_{0}+\rho G_{r} \Theta_{0}=0
\end{gathered}
$$




$$
\left(\nabla^{2}-N^{2}\right) \Theta_{0}=0
$$

for the order- $O\left(\mathrm{f}_{0}\right)$ equations and

$$
\begin{gathered}
\rho \frac{\partial u_{1}}{\partial r}+\frac{2 \rho}{r} u_{1}+u_{1} \frac{\partial \rho}{\partial r}+\frac{\rho}{r} \frac{\partial v_{1}}{\partial \theta}+\frac{\rho}{r} v_{1} \cot \theta=0 \\
\rho\left\{\frac{1}{r} \frac{\partial}{\partial r}\left(r\left[u_{0} \frac{\partial v_{0}}{\partial r}+\frac{v_{0}}{r} \frac{\partial v_{0}}{\partial \theta}+\frac{u_{0} v_{0}}{r}-\frac{w_{0}^{2}}{r} \cot \theta\right]\right)-\frac{1}{r} \frac{\partial}{\partial \theta}\left[u_{0} \frac{\partial u_{0}}{\partial r}+\frac{v_{0}}{r} \frac{\partial u_{0}}{\partial \theta}-\frac{v_{0}^{2}+w_{0}^{2}}{r}\right]\right\}==\left(\nabla^{2}-\chi^{2}-M^{2}-\frac{1}{r^{2} \sin ^{2} \theta}\right) \\
{\left[\frac{1}{r} \frac{\partial}{\partial r}\left(r v_{1}\right)-\frac{1}{r} \frac{\partial u_{1}}{\partial \theta}\right]+\rho G_{r}\left(\frac{\partial \Theta_{1}}{\partial r}-\frac{\partial \Theta_{1}}{\partial \theta}\right)+G_{r} \Theta_{1}\left(\frac{\partial \rho}{\partial r}+\frac{\rho}{r}\right)} \\
\rho\left[u_{0} \frac{\partial w_{0}}{\partial r}+\frac{v_{0}}{r} \frac{\partial w_{0}}{\partial \theta}+\frac{u_{0} w_{0}}{r}+\frac{v_{0} w_{0}}{r} \cot \theta\right]=\left(\nabla^{2}-\chi^{2}-M^{2}-\frac{1}{r^{2} \sin ^{2} \theta}\right) w_{1}+\rho G_{r} \Theta_{1} \\
\rho \operatorname{Pr}\left[u_{0} \frac{\partial \Theta_{0}}{\partial r}+\frac{v_{0}}{r} \frac{\partial \Theta_{0}}{\partial \theta}\right]=\left(\nabla^{2}-N^{2}\right) \Theta_{1}
\end{gathered}
$$

for the order- $O\left(\mathrm{f}_{1}\right)$ equations.

The solutions to the flow variables in the orders $O\left(f_{0}\right)$ and $O\left(f_{l}\right)$ equations can be effected by starting with the temperature equations and using the following transformations;

$$
\begin{gathered}
\Theta_{0}(r, \theta)=\Theta_{00}(r) \sin \theta \\
w_{0}(r, \theta)=w_{00}(r) \sin \theta \\
u_{0}(r, \theta)=u_{00}(r) \cos \theta \\
v_{0}(r, \theta)=v_{00}(r) \sin \theta \\
\Theta_{1}(r, \theta)=\Theta_{10}(r) \sin 2 \theta \\
w_{1}(r, \theta)=w_{10}(r) \sin 2 \theta \\
u_{1}(r, \theta)=u_{10}(r)\left(2 \cos ^{2} \theta-\sin ^{2} \theta\right)
\end{gathered}
$$

and

$$
v_{1}(r, \theta)=v_{10}(r) \sin 2 \theta .
$$

The solutions to the flow variables, obtained by the

$$
\begin{aligned}
& \Theta_{0}(r)=a_{1} i_{1}(N r)+a_{2} k_{1}(N r) \\
& w_{0}(r)=b_{1} i_{1}(\beta r)+b_{2} k_{1}(\beta r)-\frac{G_{r}}{\beta} \int \rho(r) \Theta_{0}(r) d r(24) \\
& f_{1}(r)=\frac{\rho(r)}{2 \beta^{2}}\left(\left[u_{0}(r) \frac{d w_{0}(r)}{d r}+\frac{u_{0}(r) w_{0}(r)}{r}+\frac{2 v_{0}(r) w_{0}(r)}{r}\right]-2 G_{r} \Theta_{1}(r)\right) h_{1}(r)=-3 \psi_{1}(r), \\
& \psi_{1}(r)=c_{11} i_{2}(\beta r)+c_{22} k_{2}(\beta r)+\beta \int Y_{1}(r) d r, \\
& \psi_{0}(r)=c_{1} \dot{i}_{1}(\beta r)+c_{2} k_{1}(\beta r)+\beta \int Y_{0}(r) d r, \\
& Y_{0}(r)=-\frac{1}{\beta^{2}}\left\{\rho(r) G_{r} \frac{d \Theta_{0}(r)}{d r}+G_{r} \Theta_{0}(r)\left(\frac{d \rho}{d r}+\frac{\rho}{r}\right)\right\}, \\
& T_{1}(r)=\frac{\rho(r) \operatorname{Pr}}{2 N^{2}}\left[u_{0}(r) \frac{d \Theta_{0}(r)}{d r}+\frac{v_{0}(r) \Theta_{0}(r)}{r}\right] .
\end{aligned}
$$

and

$$
v_{1}(r)=-d_{21} r+\frac{2}{3} d_{22} r^{-4}-\frac{r}{5} \int r^{-1} h_{1}(r) d r-\frac{2 r^{-4}}{15} \int r^{4} h_{1}(r) d r
$$

where, $z=N r$ and $\eta=\beta r$ and $i_{n}(z), k_{n}(z), i_{n}(\eta)$ and $k_{n}(\eta)$ are respectively the modified spherical Bessel functions of the first and second kind of order $n$, with $z$ and $\eta$ as the arguments. Whereas, 
and

$$
\begin{aligned}
Y_{1}(r)=\frac{\rho(r)}{2 \beta^{2}} & \left\{\frac{1}{r} \frac{\partial}{\partial r}\left(r u_{0}(r) \frac{d v_{0}(r)}{d r}+v_{0}^{2}(r)+u_{0}(r) v_{0}(r)-w_{0}^{2}(r)\right)+\left(\frac{2 u_{0}(r)}{r} \frac{d u_{0}(r)}{d r}+\frac{2 v_{0}(r) u_{0}(r)}{r^{2}}+\frac{2 v_{0}{ }^{2}(r)}{r^{2}}+\frac{2 w_{0}{ }^{2}(r)}{r^{2}}\right)\right\} \\
- & \frac{G_{r}}{\beta^{2}}\left[\rho(r) \frac{d \Theta_{1}(r)}{d r}+\Theta_{1}(r)\left(\frac{d \rho(r)}{d r}+\frac{\rho(r)}{r}\right)\right]
\end{aligned}
$$

with

$$
\begin{gathered}
\beta^{2}=\left(\chi^{2}+M^{2}\right), \\
a_{1}=\frac{\Theta_{0}\left(r_{1}\right) k_{1}\left(N r_{2}\right)-\Theta_{0}\left(r_{2}\right) k_{1}\left(N r_{1}\right)}{i_{1}\left(N r_{1}\right) k_{1}\left(N r_{2}\right)-i_{1}\left(N r_{2}\right) k_{1}\left(N r_{1}\right)}, a_{2}=\frac{\Theta_{0}\left(r_{2}\right) i_{1}\left(N r_{1}\right)-\Theta_{0}\left(r_{1}\right) i_{1}\left(N r_{2}\right)}{i_{1}\left(N r_{1}\right) k_{1}\left(N r_{2}\right)-i_{1}\left(N r_{2}\right) k_{1}\left(N r_{1}\right)}, b_{1}=\frac{f_{0}\left(r_{1}\right) k_{1}\left(\beta r_{2}\right)-f_{0}\left(r_{2}\right) k_{1}\left(\beta r_{1}\right)}{i_{1}\left(\beta r_{1}\right) k_{1}\left(\beta r_{2}\right)-i_{1}\left(\beta r_{2}\right) k_{1}\left(\beta r_{1}\right)}, \\
b_{2}=\frac{f_{0}\left(r_{1}\right) k_{1}\left(\beta r_{2}\right)-f_{0}\left(r_{2}\right) k_{1}\left(\beta r_{1}\right)}{i_{1}\left(\beta r_{1}\right) k_{1}\left(\beta r_{2}\right)-i_{1}\left(\beta r_{2}\right) k_{1}\left(\beta r_{1}\right)}, \\
d_{1}=\frac{u_{0}\left(r_{2}\right) r_{1}^{-3}-u_{0}\left(r_{1}\right) r_{2}^{-3}}{r_{2}^{-3}-r_{1}^{-3}}, d_{2}=\frac{u_{0}\left(r_{1}\right)-u_{0}\left(r_{2}\right)}{r_{2}^{-3}-r_{1}^{-3}}, d_{11}=\frac{v_{0}\left(r_{1}\right) r_{1}^{3}-v_{0}\left(r_{2}\right) r_{2}^{3}}{r_{2}^{3}-r_{1}^{3}}, \\
d_{12}=\frac{2 r_{1}^{3} r_{2}^{3}\left\{v_{0}\left(r_{1}\right)-v_{0}\left(r_{2}\right)\right\}}{r_{2}^{3}-r_{1}^{3}}, a_{11}=\frac{\left(\Theta_{1}\left(r_{1}\right)-T_{1}\left(r_{1}\right)\right) k_{2}\left(N r_{2}\right)-\left(\Theta_{1}\left(r_{2}\right)-T_{1}\left(r_{2}\right)\right) k_{2}\left(N r_{1}\right)}{i_{2}\left(N r_{1}\right) k_{2}\left(N r_{2}\right)-i_{2}\left(N r_{2}\right) k_{2}\left(N r_{1}\right)}, \\
a_{22}=\frac{\left(\Theta_{1}\left(r_{2}\right)-T_{1}\left(r_{2}\right)\right) i_{2}\left(N r_{1}\right)-\left(\Theta_{1}\left(r_{1}\right)-T_{1}\left(r_{1}\right)\right) i_{2}\left(N r_{2}\right)}{i_{2}\left(N r_{1}\right) k_{2}\left(N r_{2}\right)-i_{2}\left(N r_{2}\right) k_{2}\left(N r_{1}\right)}, \\
b_{11}=\frac{f_{1} s\left(r_{2}\right) k_{2}\left(\beta r_{1}\right)-f_{1} s\left(r_{1}\right) k_{2}\left(\beta r_{2}\right)}{i_{2}\left(\beta r_{1}\right) k_{2}\left(\beta r_{2}\right)-i_{2}\left(\beta r_{2}\right) k_{2}\left(\beta r_{1}\right)}, b_{22}=\frac{f_{1} s\left(r_{1}\right) i_{2}\left(\beta r_{2}\right)-f_{1} s\left(r_{2}\right) i_{2}\left(\beta r_{1}\right)}{i_{2}\left(\beta r_{1}\right) k_{2}\left(\beta r_{2}\right)-i_{2}\left(\beta r_{2}\right) k_{2}\left(\beta r_{1}\right)}, \\
d_{10}=\frac{u_{1} s\left(r_{2}\right) r_{1}^{-4}-u_{1} s\left(r_{1}\right) r_{2}^{-4}}{r_{1} r_{2}^{-4}-r_{2} r_{1}^{-4}}, d_{20}=\frac{u_{1} s\left(r_{1}\right) r_{2}-u_{1} s\left(r_{2}\right) r_{1}}{r_{1} r_{2}^{-4}-r_{2} r_{1}^{-4}}, \\
d_{21}=\frac{v_{1} s\left(r_{1}\right) r_{1}^{4}-v_{1} s\left(r_{2}\right) r_{2}^{4}}{r_{2}^{5}-r_{1}^{5}}
\end{gathered}
$$

and

$$
d_{22}=\frac{3 r_{1}^{4} r_{2}^{4}\left\{v_{1} s\left(r_{1}\right) r_{2}-v_{1} s\left(r_{2}\right) r_{1}\right\}}{2\left(r_{2}^{5}-r_{1}^{5}\right)} .
$$

While, $r_{1}$ and $r_{2}$ are respectively the non-dimensional radii at the boundaries and the other constants that emanated from the solutions of the equations are given as follows;

$$
\begin{gathered}
c_{1}=\frac{Y_{0}\left(r_{1}\right) k_{1}\left(\beta r_{2}\right)-Y_{0}\left(r_{2}\right) k_{1}\left(\beta r_{1}\right)}{i_{1}\left(\beta r_{1}\right) k_{1}\left(\beta r_{2}\right)-i_{1}\left(\beta r_{2}\right) k_{1}\left(\beta r_{1}\right)} \\
c_{2}=\frac{Y_{0}\left(r_{1}\right) k_{1}\left(\beta r_{2}\right)-Y_{0}\left(r_{2}\right) k_{1}\left(\beta r_{1}\right)}{i_{1}\left(\beta r_{1}\right) k_{1}\left(\beta r_{2}\right)-i_{1}\left(\beta r_{2}\right) k_{1}\left(\beta r_{1}\right)} \\
c_{11}=\frac{Y_{1}\left(r_{2}\right) k_{2}\left(\beta r_{1}\right)-Y_{1}\left(r_{1}\right) k_{2}\left(\beta r_{2}\right)}{i_{2}\left(\beta r_{1}\right) k_{2}\left(\beta r_{2}\right)-i_{2}\left(\beta r_{2}\right) k_{2}\left(\beta r_{1}\right)}
\end{gathered}
$$




$$
c_{22}=\frac{Y_{1}\left(r_{1}\right) i_{2}\left(\beta r_{2}\right)-Y_{1}\left(r_{2}\right) i_{2}\left(\beta r_{1}\right)}{i_{2}\left(\beta r_{1}\right) k_{2}\left(\beta r_{2}\right)-i_{2}\left(\beta r_{2}\right) k_{2}\left(\beta r_{1}\right)}
$$



Figure 1. Temperature profiles for various values of Radiation Parameter $\left(N^{2}\right)$.

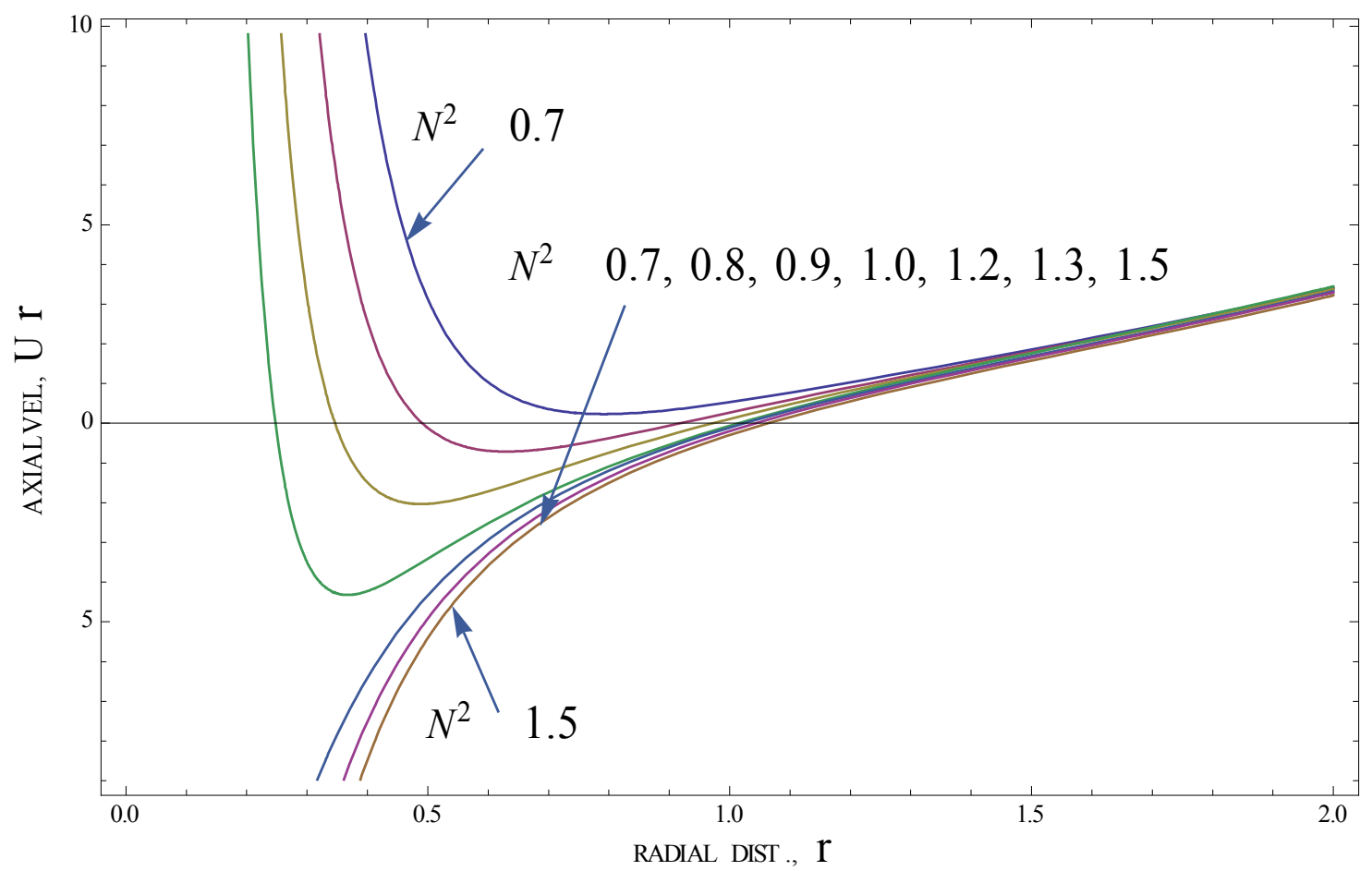

Figure 2. Axial Velocity profiles for various Radiation Parameter $\left(N^{2}\right)$.

\section{Discussion}

The primary aim of this study is to investigate the effect of density variation on the dynamical variables, such as temperature and velocity; of a plasma gas in a radiating and slowly rotating hot sphere. To this end, Section 2 presents the mathematical model of the problem incorporating the density as an exponential function of the radial distance, $r$. The results of the analysis using the Wolframs Mathematica software (version 9) are presented in Figures 1 to 7 for the following realistic computational parameters; $\left(\varepsilon_{0}=0.0,0.1,0.3,0.5,0.7\right.$, 
1.0, 2.0, 5.0 and 10.0); $\left(N^{2}=0.4,0.5,0.7,0.8,0.9,1.0,1.1\right.$, $1.2,1.3$ and 1.5$) ;\left(M^{2}=0.6,0.7,0.8,0.9,1.0,1.2,1.4,1.6,1.8\right.$ and 2.0$) ;(\operatorname{Re}=0.001,0.01,0.1)$ and $(\operatorname{Pr}=0.71)$.

The analysis shows that variation of the plasma density has tremendous effects on the profiles of the plasma flow variables. For example, the temperature of the plasma is seen to decease to a minimum value at the point where $r=1.4 R_{\circledast}$ and then suddenly increase to a maximum value when $r=3.5$ $R_{\otimes}$ from where it decreases as $r$ tends to infinity $(r \rightarrow \infty)$.
Similar observation is noticed in the case of the velocity profiles (Figure 2). On the other hand, the model shows that the flow experience a velocity reversal when the magnetic field parameter is greater than 0.8 (i.e., $M^{2}>0.8$ ). That is, the magnitude of the flow velocities according to Figures 1, 5 and 6 increase with magnetic field strength and thermal radiation parameter but decreases with increase in the density parameter. The general picture of the temperature distribution of the flow model is presented in 3D format in Figure 7.

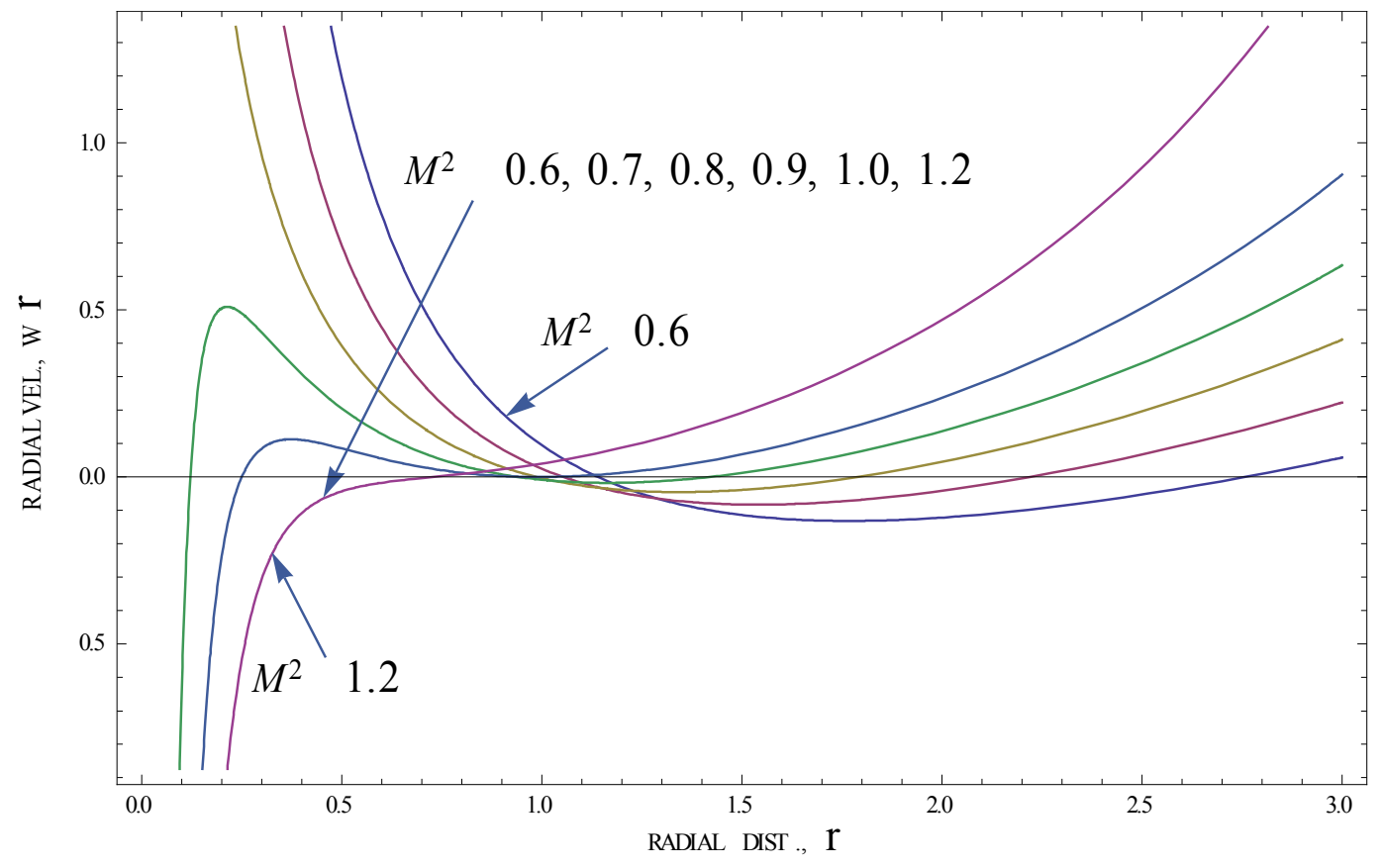

Figure 3. Radial Velocity profiles for various values of Magnetic Parameter $\left(M^{2}\right)$.

The region with low ions density indicates that it is dominated by the neutral atoms. In this case, the flow experience ambipolar effects. This is seen to account for the reduction in the velocity of the plasma. [27 - 30] suggest that this is true in the case of the $D$ and $E$ layers of the ionosphere.

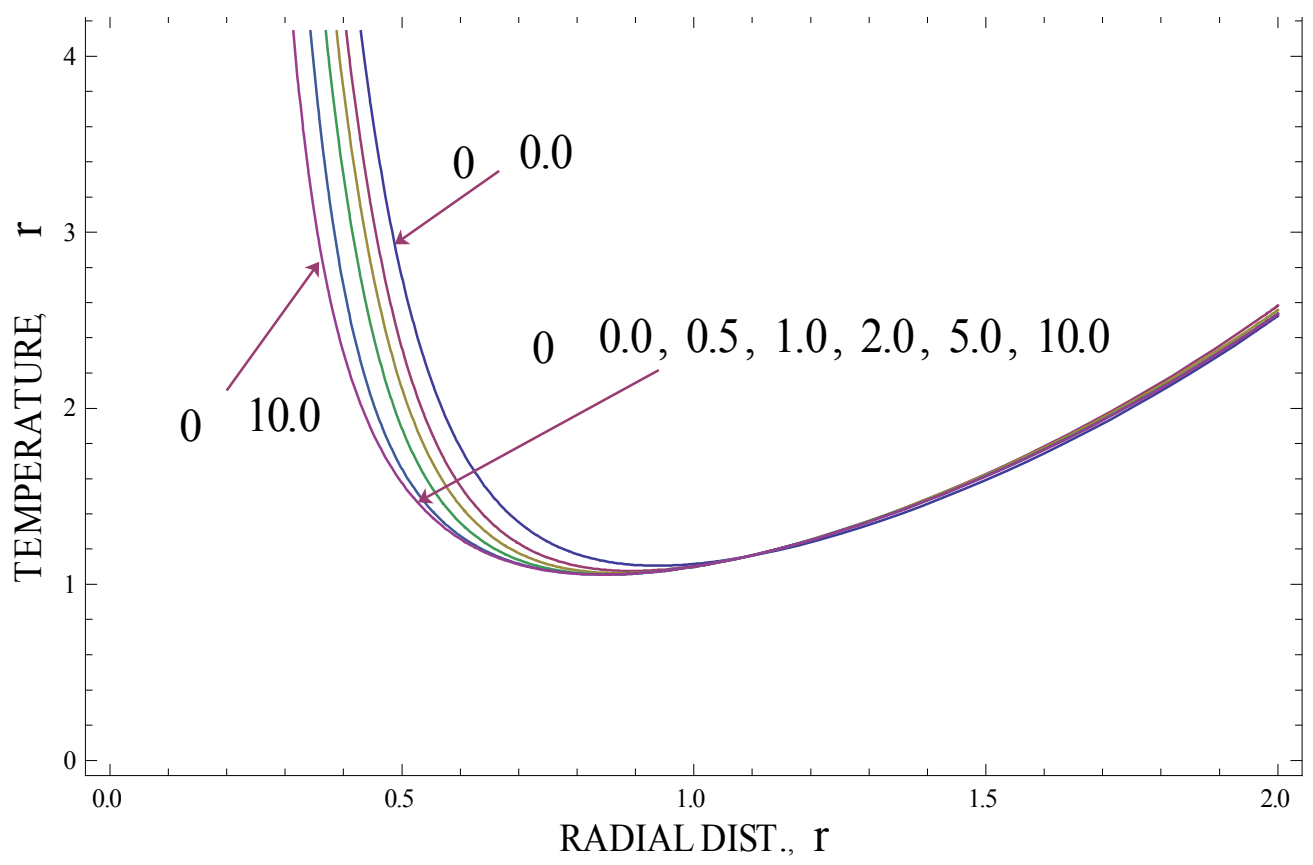

Figure 4. Temperature profiles for various values of Density Parameter $\left(\varepsilon_{0}\right)$. 
It is observed that when the density parameter, $\varepsilon_{0}$ is low the fluid tends to be populated by ions and electrons and their interactions lead to increase in the kinetic energy of the plasma and a subsequent increase in the temperature as can be seen from Figure 7. [20, 31 - 33] indicate that such high temperature and low pressure within the gas lead to gas rarefication, resulting in the production of rarefaction waves which flow in the reverse direction. The reversal carries along with it energetic particles at high temperature resulting in the heating up of the upper solar atmosphere [12, 34]. Generally, the analysis aids our understanding of astrophysical interiors and its atmospheres as well as the interactions in the $D$ and $E$ layers of the Earth's ionosphere as noted by [35].

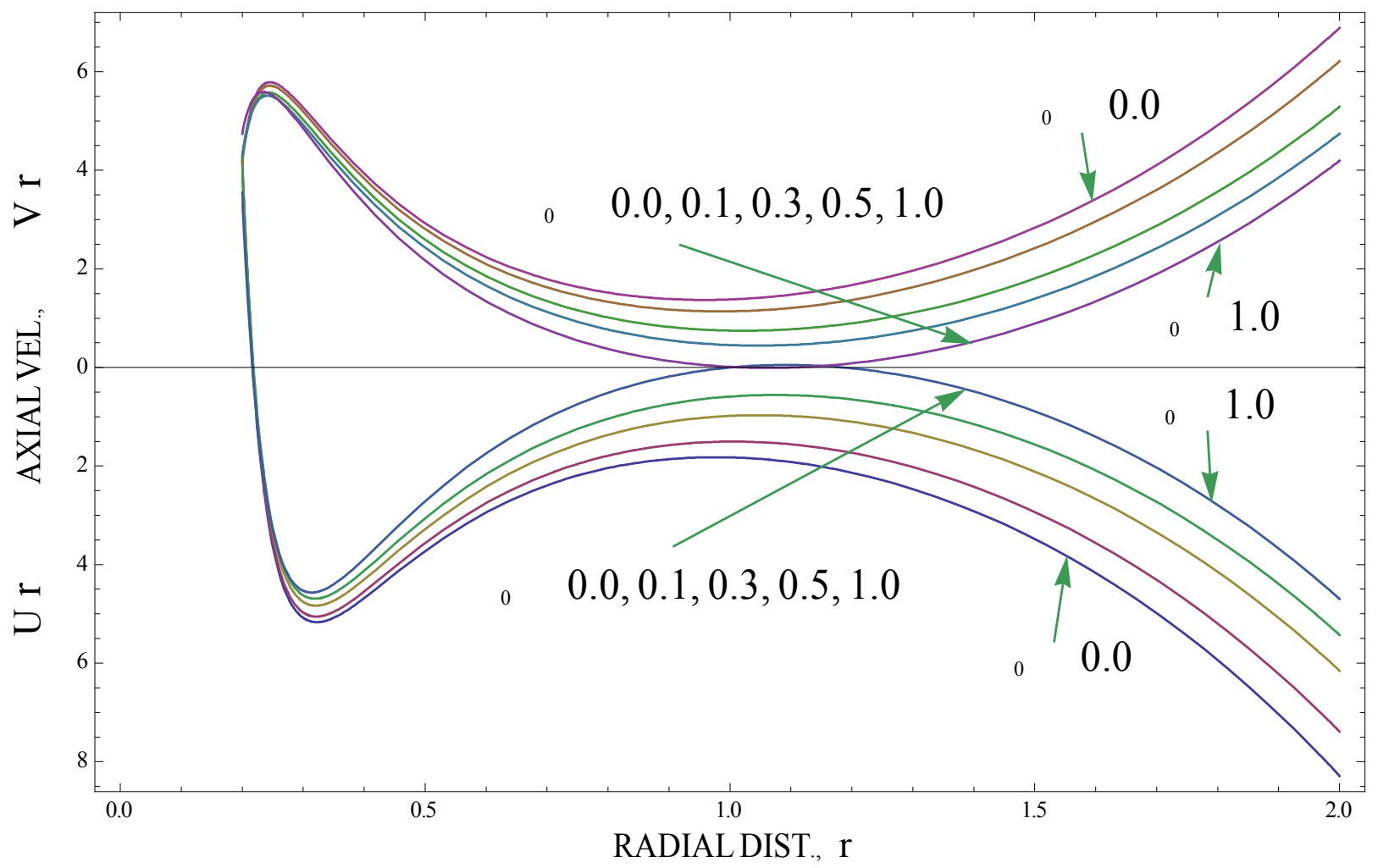

Figure 5. Axial Velocity profiles for various values of Density Parameter $\left(\varepsilon_{0}\right)$.



Figure 6. Axial Velocity profiles for various Magnetic Parameter $\left(M^{2}\right)$. 


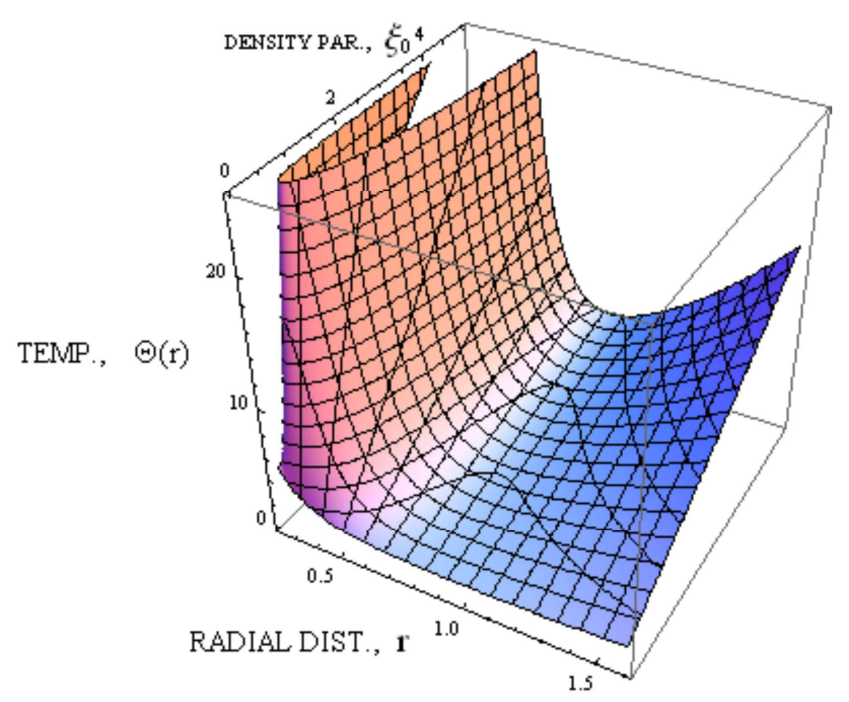

Figure 7. $3 D$ Temperature profile with Density Parameter $\left(\varepsilon_{0}\right)$.

\section{Conclusion}

The rarefaction waves produced by the interactions of ionized species of the plasma at low density parameters lead to flow reversal. Energetic fluid elements carried along in this reversed flow heat up the plasma which could account for the high temperatures observed in upper solar atmosphere. The reduction in temperature above the solar sphere leads to increased presence of neutral atoms in the lower chromosphere. In this case, the flow experience ambipolar effects resulting from the interactions of the ionized species with the neutrals [35]. Generally, variation in the plasma density is seen to have an important effect on these interactions and the flow profiles of the plasma.

\section{References}

[1] Abbey, T. M. (1996); The flow of a two-component plasma gas model past a porous rotating hot sphere, Nig. J. Phys. 8S: $51-60$.

[2] Abbey, T. M. and Mbeledeogu, I. U. (1998); Hydrodynamic slip flow of a radiating fluid with Hall current, Part II: Fully developed flow with axial temperature and concentration variation, Int. J. Energy Res., 22: 93-105.

[3] Abbey, T. M. and John E. (2000); Transient slip flow in a twocomponent Plasma model with Radiative heat transfer, J. Math. Sci. Forum 2: 37-47.

[4] Alagoa, K. D. and Abbey, T. M. (2001); Temperature distribution in the Solar globe due to exponentially varying plasma density, J. Math. Sci. Forum 3: 1-8.

[5] Israel - Cookey, C., Amos, E. and Nwaigwe, C. (2010); MHD oscillatory Couette flow of a radiating viscous fluid in a porous medium with periodic wall temperature, Am. J. Sci. Ind. Res., 1(2): 326-331.

[6] Sanatan, D., Mrinal, J. and Rabindra, N. J. (2011); Radiation effect on natural convection near a vertical plate embedded in porous medium with ramped wall temperature, Open $J$. of Fluid Dynamics, 1:1-11.

[7] Sherman, F. S. (1990); Viscous flow; flow with nearly constant density and transport properties, International edition, McGraw-Hill.

[8] Badalyan, O. G. (1986); Polarization of white-light corona under hydrostatic density distribution" Astron. and Astrophysics, 168 (1-2): 305-312.

[9] Arthur, N. C., Williams, C. L. and Mildred, S. M. (1991); Solar interior and atmosphere, University of Arizona Press.

[10] Badalyan, O. G. and Livshits, M. A. (1986); The K-corona under hydrostatic density distribution relevance to solar wind" Solar Physics, 103(2): 385-392.

[11] Badalyan, O. G. (1988); Evidence of coronal expansion from data on the electron component, Sov. Astron. 32(2), 205-210.

[12] Priest, E. R. (1982); Solar magneto-hydrodynamics 1, D. Reidel Publishing Company, Holland.

[13] Alagoa, K. D. and Sakanaka P. H. (1998); Gravitational stability of the solar Globe, ICTP: IC/98/112.

[14] Ko, M. (1999); Density of the Sun, In Elert, G. The Physics Factbook.

[15] Zirker, J. B. (2002); Journey from the Center of the Sun, Princeton University Press, 11: 119-127.

[16] Williams, D. R. (2013); Sun Fact Sheet, NASA. Retrieved 2013-08-12.

[17] Bestman A. R. (1988); Unsteady low Reynolds number flow in a heated tube of slowly varying section, J. Austral. Math. Soc. Ser. B. 30: 179-202.

[18] Abbey, T. M., Bestman, A. R. and Mbeledeogu, I. U. (1992); Flow of a two-component plasma model in a porous rotating hot sphere, Astrophysics and Space Sci., 197: 61-76.

[19] Cheng, P. (1964); Two-Dimensional radiating gas flow by moment method, AIAAJ 21662.

[20] Abbey, T. M. and Bestman, A. R. (1995); Slip flow in a twocomponent plasma model with radiative heat transfer" Int. J. Energy Research, 19: 1-6.

[21] Bestman A. R. (1983); Low Reynolds number flow in a heated tube of varying section, J. Austral. Math. Soc. Ser. B. 25: 244260.

[22] Alagoa, K. D., Tay, G. and Abbey, T. M. (1999); Radiative and convective Effects of a MHD flow through a porous medium sandwiched between two infinite parallel plates with time dependent suction, Astrophysics and Space Sci., 260: 455468.

[23] Idowu, A. S., Joseph, K. M. and Daniel, S. (2013); Effect of heat and mass transfer on unsteady MHD oscillatory flow of Jeffrey fluid in a horizontal a channel with chemical reaction, IDSR Journal of Mathematics (idsr-jm), 8(5): 74-87.

[24] Eid, A. (2014); Dynamics of a radiating thick shell, Adv. Studies Theor. Phys. 8(4): 163-168.

[25] Abramowitz, M. and Stegun, I. A. (1972); Handbook of mathematical functions, with formulas, graphs and mathematical tables, Tenth Printing, Dover; New York.

[26] Olver, F. W. J. (2010); NIST Handbook of mathematical functions, Cambridge University Press, USA.

[27] Elmegreen, B. G. (1979); Astrophysical Journal, 232: 729. 
[28] Myers, P. C. (1985); Protostars and Planets II, ed. M. S. Matthews and D. C. Black (Tucson, AZ: Univ. Arizona Press), 81. GmbH \& Co. KGaA, Weinheim.

[29] Van Loo, S., Falle, S. A. E., Hartquist, T. W. and Barker, A. J. (2008); The effect of ambipolar resistivity on the formation of dense cores, Astron. and Astrophysics manuscript no. version 2 .

[30] Choi, E., Kim, J. and Wiita, P. J. (2009); An explicit scheme for incorporating ambipolar diffusion in a magnetohydrodynamics code, The Astrophysical J. Supplement Series 181: 413.

[31] Street, R. E. (1960); Rarefied gas dynamics, Pergamon Press.
[32] Ram, P. C. (1988); Hall effect on the hydromagnetic free convective flow and mass transfer through a porous medium bounded by an infinite vertical porous plate with constant heat flux, Int. J. Energy Research, 12: 227-231.

[33] Ram, P. C. (1990); MHD convective flow of a viscous heat generating fluid in slip flow regime with Hall currents, Int. J. Energy Research, 14: 465-467.

[34] Woods, L. C. (2004); Physics of plasma, Wiley-VCH, Verlag.

[35] Tuduo, B. S. and Abbey, T. M. (2017); Effect of ambipolar diffusion on the flow of a two-component plasma gas model in the Earth's planetary ionosphere, International Journal of Astrophysics and Space Science. 5(3): 47-54. 